Editorial

\title{
When less is more: Gaining power through gene rearrangement of amplified EGFR
}

\section{Tomoyuki Koga, Clark C. Chen and Frank B. Furnari}

Extrachromosomal gene amplification refers to copy number gains of DNA fragments that are not contiguous with the twenty-three canonical chromosomes. Unlike typical chromosomes, they are composed of circular DNA fragments without centromeric sequences [1]. They arose through complex genomic rearrangements during carcinogenesis and commonly harbor oncogenes, conferring selective advantage for tumor growth and survival [2]. Such extrachromosomal elements, often referred to as double minutes, are frequently observed throughout different types of cancer, especially in glioblastoma [3]. They are dynamically regulated and play important roles in acquired therapeutic resistance [4] and clonal evolution [3].

Epidermal growth factor receptor gene (EGFR) is, by far, the most commonly altered oncogene in glioblastomas, the most common form of primary brain cancer. It is mutated or amplified in $60 \%$ of glioblastomas [5]. Oncogenic activation of EGFR appears to preferentially occur through deletion of exons two to seven, generating a constitutively activated receptor termed EGFRvIII. Up to $30 \%$ of glioblastomas harbor
EGFRvIII. Importantly, EGFRvIII is always accompanied by amplification of the full-length EGFR gene. EGFRvIIIpositive glioblastomas exhibit unique molecular physiologies associated with distinct therapeutic response profiles and an overall tumor aggressiveness [6].

Breakpoint junctions in EGFRvIII are highly variable between tumor samples due to 120 -kb-long intron one of this gene where one of the breaks take place. Breakpoint sequence analyses implicate contribution by multiple DNA strand-break repair mechanisms, including non-homologous end joining, micro-homology mediated end joining, and other forms of replication mediated repair [7]. These findings suggest DNA strand breaks as a likely intermediate structure for the EGFR deletion variants.

Another interesting observation pertaining to EGFRvIII is that a single tumor can harbor multiple, independent EGFRvIII variants -- each with distinct breakpoint junctions. These variants as well as the accompanied amplified full-length EGFR share common allelic profiles, suggesting that the deleted variants arose from a common ancestral amplified EGFR. A corollary of this hypothesis is that distinct additional

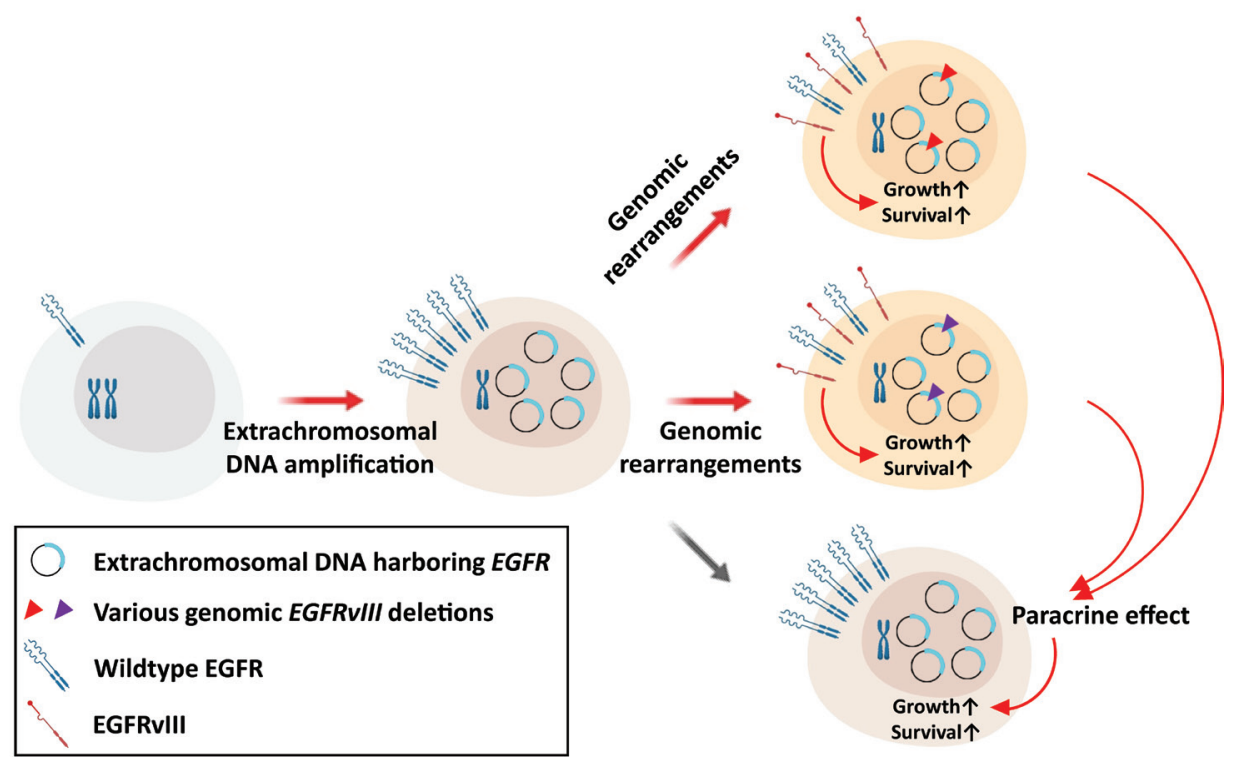

Figure 1: The possible mechanisms of tumor progression through genomic rearrangements of extrachromosomal DNA in glioblastoma. Models for generation of heterogeneous EGFRvIII breakpoints through genomic rearrangements. Cells with extrachromosomal amplifications of EGFR undergo further independent genomic rearrangements resulting in heterogeneous populations expressing EGFRvIII from differentially edited EGFR genes. Those cells acquiring EGFRvIII are not only enhanced for aggressive tumor growth but also benefit surrounding cells without the $E G F R$ mutation through paracrine effect. 
genomic rearrangements of amplified full-length $E G F R$ in glioblastoma subpopulations gave rise to polyclonal EGFRvIII breakpoint species (Figure 1).

An important mechanistic question in this context involves the sequence of events that led to $E G F R$ variant formation and $E G F R$ amplification. Is extrachromosomal $E G F R$ amplification a requisite precursor of EGFR IIII? For instance, this acquisition of glioblastoma specific $E G F R$ deletion mutation might be associated with the fact that amplified EGFR in glioblastoma mostly resides in extrachromosomal DNA that is selectively captured in micronuclei [1], where complex DNA rearrangements take place [8]. In this way, extrachromosomal DNA generation possibly provides a path for the host cell to enhance malignant progression through micronuclei-mediated genomic rearrangements resulting in EGFRvIII.

Once EGFRvIII is formed, not only do cells expressing this mutant transform to a more aggressive phenotype as mentioned above, but surrounding tumor cells without mutant EGFR are also benefited in terms of growth and cell survival by paracrine effects through cytokines such as interleukin-6 [9], or through EGFR ligands such as heparin-binding EGF and transforming growth factor alpha [10] (Figure 1). Thus, tumors with subpopulations undergoing genomic rearrangements of amplified EGFR yielding EGFRvIII that establishes an ecosystem supportive of aggressive tumor growth. Elucidation of this mechanisms of further rearrangements of extrachromosomal amplicons should yield opportunities for future therapeutic development and mechanistic insight.

Frank B. Furnari: Ludwig Cancer Research, University of California San Diego, La Jolla, CA, USA

Correspondence to: Frank B. Furnari,

email ffurnari@ucsd.edu

Keywords: genetic rearrangement; mutation; oncogene amplification

Received: February 26, 2019

Published: March 15, 2019

\section{REFERENCES}

1. Shimizu N, et al. Nat Genet. 1996; 12:65-71.

2. Holland AJ, Cleveland DW. Nat Med. 2012; 18:1630-8.

3. Turner KM, et al. Nature. 2017; 543:122-5.

4. Nathanson DA, et al. Science. 2014; 343:72-6.

5. Brennan CW, et al. Cell. 2013; 155:462-77.

6. Nishikawa R, et al. Proc Natl Acad Sci U S A. 1994; 91:7727-31.

7. Koga T, et al. Neuro Oncol. 2018; 20:1310-20.

8. Hatch EM, Hetzer MW. Cell. 2015; 161:1502-4.

9. Zanca C, et al. Genes Dev. 2017; 31:1212-27.

10. Ramnarain DB, et al. Cancer Res. 2006; 66:867-74.

Copyright: Koga et al. This is an open-access article distributed under the terms of the Creative Commons Attribution License 3.0 (CC BY 3.0), which permits unrestricted use, distribution, and reproduction in any medium, provided the original author and source are credited. 